\title{
Nuevas generaciones y empleo. Características psico-sociales de las generaciones Z y su inserción en las estructuras organizacionales asociadas al empleo (CABA, 2016)
}

\author{
Dr. Esteban Maioli ${ }^{1}$ \\ INSOD - UADE
}

Mg. Marina Filipuzzi

INSOD - UADE

\begin{abstract}
Artículo científico
Material original autorizado para su primera publicación en el Journal de Ciencias Sociales, Revista Académica de la Facultad de Ciencias Sociales de la Universidad de Palermo.
\end{abstract}

\section{Recibido: 10-10-2016}

Aceptado: 18-12-2016

Resumen: La Generación Z es entendida como el grupo poblacional que, en virtud de su fecha de nacimiento (hacia 1995 en adelante), cuenta con características psico-sociales específicas, que la diferencian de las generaciones históricas previas. El artículo pretende mostrar algunas de las características sobresalientes de la nueva generación (la última en ser reconocida por la literatura especializada), al mismo tiempo que resaltar los aspectos que deberán ser considerados por las estructuras organizacionales asociadas al "mundo del trabajo" para poder reclutar y retener a estos jóvenes talentos.

Palabras clave: Generación Z, Características psico-sociales, organizaciones, empleo, expectativas.

Abstract: People borned since 1995 are called the $Z$ generation. This generation presents particular psycosocial specific characteristics. This article shows some of the relevant characteristics of this generation trying to highlight the aspects that have to be considered for the organizations dedicated to the labor market in order to call up and contain young talents.

Key words: Generation Z, Psychosocial characteristics, organizations, employment, expectations.

\footnotetext{
${ }^{1}$ Doctor en Ciencias Sociales, FLACSO, Argentina. Docente e Investigador en la Universidad Argentina de la Empresa. Correo electrónico: estebanmaioli@live.com.ar

${ }^{2}$ Magíster en Dirección de Recursos Humanos. Docente e Investigadora en la Universidad Argentina de la Empresa. Correo electrónico: mfilipuzzi@uade.edu.ar
} 


\section{Introducción}

Los estudios generacionales cuentan con una importante tradición de investigación en el conjunto de las ciencias sociales y humanas. Las primeras investigaciones realizadas por el sociólogo alemán Karl Mannheim (1928) iniciaron una fructífera línea de indagación científica sobre las generaciones históricas.

Por un lado, la celeridad del cambio social que se advierte en el contexto de las sociedades en contexto de globalización impone la necesidad de indagar sobre las transformaciones que son advertidas tanto en la subjetividad de los agentes involucrados como de su inserción en ámbitos institucionales de relevancia social, tales como el empleo. En este sentido, la Generación Z, entendida como el agrupamiento poblacional nacido hacia fines del siglo XX e inicios del siglo XXI, presentan características exclusivas, diferentes de las generaciones previas, y que resultan imprescindibles conocer para revisar los impactos que tales cualidades tendrán en el ámbito institucional del empleo.

Por otro lado, Smola y Sutton (2002) sostienen que los gerentes de organizaciones frecuentemente tienen que lidiar con las diferencias generacionales que parecen existir entre los trabajadores. La literatura especializada es rica en el reconocimiento de tales diferencias. En la actualidad, las organizaciones cuentan con integrantes que pertenecen a diferentes generaciones (baby boomers, generación $\mathrm{X}$, generación $\mathrm{Y}$ o millenials, y los recientemente identificados como generación Z). De acuerdo con Addor (2011), nunca antes las organizaciones habían enfrentado el desafío de contar con un grupo tan heterogéneo de miembros. Los miembros de cada generación histórica se caracterizan por un conjunto diferente de valores, creencias, actitudes y ambiciones que se hacen manifiestas en su acción concreta en las estructuras organizacionales.

Al mismo tiempo, muchas veces las organizaciones asumen como reales ciertas cualidades de los integrantes de las nuevas generaciones que se encuentran fundadas en estereotipos o representaciones sociales que no encuentran su correlato con la realidad. En esta línea, muchas veces se afirman acciones asumidas por las organizaciones que darían cuenta de un entendimiento de las expectativas de las nuevas generaciones sobre el mundo del trabajo que no encuentran una real concretización en la práctica y cultura organizacional. Sin dudas, entender las diferencias generacionales puede ser una herramienta para los gerentes de organizaciones para crear ambientes de mayor productividad, innovación y actitud de lealtad corporativa.

Este artículo se funda en la reflexión y análisis de una investigación empírica conducida en el ámbito urbano de la Ciudad Autónoma de Buenos Aires, Argentina, durante el año 2016. Recupera las expectativas de los miembros de la generación Z con relación al "mundo del trabajo" y su incorporación en organizaciones, al tiempo que revela las principales características psico-sociales de esta generación histórica. Pretende, al mismo tiempo, identificar las 
tensiones existentes entre las expectativas de inserción laboral de los integrantes de la generación Z y las representaciones sociales sobre tal generación que circulan entre los decisores de las estructuras organizacionales.

\section{Los estudios generacionales}

Los estudios sobre generaciones sociales son cada día más extensos, aunque limitados cuando se los compara con los que han generado otros conceptos más utilizados como el de "clase social". Sin embargo, el concepto sociológico de "generación" puede ser muy aplicable a casos concretos, y de mucha utilidad y poder explicativo cuando se lo utiliza correctamente. Como menciona Cherrington (1997):

El concepto de clase social es regularmente usado en el estudio de distintos grupos sociales por su poder de explicación y por ser relativamente familiar a los sociólogos. Con mayor aplicación a casos concretos, el concepto de "generación" puede iluminar varios aspectos del comportamiento social (p. 303).

En primer lugar, es necesario definir el término "generación social", o simplemente "generación". Una generación, según Smola y Sutton (2002), es un grupo identificable de población que comparte años de nacimiento y significativos eventos de la vida en críticas etapas de desarrollo. Son aquellos quienes comparten experiencias históricas o sociales, cuyos efectos son relativamente estables a lo largo de sus vidas. Ello se debe, fundamentalmente, al hecho de que tales eventos socio-históricos se desenvuelven durante el proceso de socialización, lo que conduce a la elaboración de valores, creencias y actitudes específicas (Berger y Luckmann, 2003). Estas experiencias tienden a distinguir una generación de la otra por afectar a grandes rasgos la forma que tienen de ver el mundo, sus valores, perspectivas y capacidades. Pilcher (1994) lo define como un agregado de personas de similar edad que involucra una participación en las mismas circunstancias históricas y sociales. Estos eventos significativos que ocurren durante la maduración, como una guerra, una época de prosperidad o de crisis económica, la innovación tecnológica, o eventos más específicos como un atentado terrorista, son importantes porque, aun inconscientemente, influyen en la formación subsecuente de las actitudes y los patrones de comportamiento de quienes están en etapa de desarrollo al momento de estos eventos. Mientras que personas de varias edades atraviesan y viven los mismos eventos, la edad a la que uno se expone a actos políticos, cambios tecnológicos o sociales, determinará cuan fuerte se instale en la psique y la cosmovisión de cada uno. Sin duda, una característica fundamental de la generación histórica son los modos compartidos de socialización, en tanto que la estructura sociocultural internalizada por los agentes miembros de una misma generación histórica es similar (aunque nunca idéntica, en virtud de la división social del trabajo y la distribución social del conocimiento). Todo ello conduce, en consecuencia, a considerar que existe una fuerte vinculación entre las características psicológicas de los miembros de una misma generación, siempre que se asume que tales características se construyen a partir de un medio social específico. 


\section{Acerca de la generación Z}

Suele considerarse a la Generación Z como el grupo poblacional cuyo nacimiento se produce después de 1995 (Wood, 2013). No obstante, no existe un pleno consenso sobre el recorte temporal de la generación. Otros especialistas explican que la Generación Z debe considerarse a partir del cambio de milenio (Bennet et al., 2012). Sea como fuese, lo cierto es que en general suele admitirse que frente a la existencia de la Generación Y (o Millenials), es posible advertir la conformación de otro agrupamiento generacional "nuevo", en virtud de las transformaciones socio-históricas que operan como contexto.

Tulgan (2013) refiere que, a diferencia de las generaciones previas, la Generación Z es la única que puede considerarse plenamente "global", en tanto que sus miembros han nacido en el contexto consolidado de la globalización. Si bien algunos especialistas sostienen que existen características psico-sociales compartidas entre la generación Y y la generación Z (Wood, 2013), es posible afirmar que, en general, se advierte que los miembros de la nueva generación cuentan con expectativas nuevas vinculadas a su inserción en la vida profesional. Cuentan, sin dudas, con habilidades específicas relacionadas con la adaptación y, fundamentalmente, con el uso de la tecnología. Al igual que la generación Z, pueden ser definidos como "multitaskers", en tanto que son capaces de realizar múltiples tareas al mismo tiempo, y en general, con el mismo grado de atención y dedicación. Lyon (2010) explica que esta habilidad es desarrollada tempranamente, principalmente debido a la gran cantidad de información que aprenden a procesar de las diversas fuentes que utilizan, todas ellas asociadas a las redes sociales y a otras tecnologías. Addor (2011) opina que, por ello, la generación Z resulta más productiva, en términos de capacidad de trabajo, que las generaciones previas. Un estudio realizado por la consultora Adecco (2015) refleja que los miembros de la generación Z cuentan con un sentimiento de confianza sobre su proyección profesional. Asimismo, explica que suelen asumir una visión del trabajo asociada con el emprendorismo (o entrepreneurship), lo cual se relaciona con un rechazo a las formas tradicionales del trabajo fuertemente regulado (en términos de horarios, condiciones, asignaciones de tareas). En general, al caracterizar a la generación $Z$, se hace referencia a la creatividad y la innovación como características sobresalientes (Half, 2015).

Algunas características sobresalientes de la Generación Z pueden ser sintetizadas del siguiente modo. Son los primeros en nacer completamente en un mundo digital. Son altamente móviles y preparados para movilizarse con suma facilidad. Con las herramientas de la comunicación al alcance de la mano, les gusta investigar todo e informarse bien, por lo que quien quiera acercarse a ellos debería mantener la información y el diálogo constantemente fluyendo, a la vez que crear espacios específicamente orientados hacia ellos, pues desean ser tenidos en cuenta. Las redes sociales son parte de su vida, siendo una buena herramienta para quien quiera acercarse a ellos. La tecnología se encuentra integrada en sus vidas. Se estima que un $75 \%$ de estos jóvenes cuenta con perfil en una red social, y que el $80 \%$ mantiene su celular cerca mientras duerme (Maioli et al, 2014). También presentan una fuerte fascinación por los videojuegos y, en general, por cualquier dispositivo tecnológico. Se muestran preocupados por los desafíos del 
mundo moderno (pobreza, desigualdad, cambio climático, entre otros). A su vez, colaboran en grupo para resolver problemas, aunque no necesariamente bajo las formas tradicionales. Las nuevas tecnologías permiten la conformación de grupos "virtuales", y generalmente son este tipo de grupos los que proliferan entre los miembros de esta generación. La Generación Z puede fácilmente conjugar la iniciativa individual con la acción comunitaria a través de sus múltiples redes sociales y la facilidad con la que se comunican. Finalmente, al vivir en un mundo más globalizado, donde los intercambios son frecuentes y es fácil comunicarse y generar acción con personas alrededor de todo el mundo, son más abiertos a la diversidad, y al mismo tiempo más adaptables. En pocas palabras, son seguros, auto-expresivos, cada vez más liberales y abiertos al cambio. Además de su clara y fuerte relación con la tecnología, presentan rasgos consumistas y de muy alta autoestima, a veces sobre-exagerada. Son optimistas, pues han crecido en tiempos de prosperidad. Además, en general son sociables, talentosos, bien educados, colaborativos, abiertos mentalmente, influyentes y orientados hacia objetivos. El uso de la tecnología, que implica acceso a dispositivos de cierto valor, provoca que asuman un carácter materialista, y que, al mismo tiempo, sean considerados algo egoístas, y orientados sólo por sus propios objetivos, con una posible sobrecarga de autoestima. Se suelen aburrir con facilidad, con períodos cortos de atención, y prefieren Internet como fuente principal de información, antes que la lectura de un libro. No les gustan las tareas rutinarias. Mientras que pueden ser buenos aprendices autodidactas, también desean el máximo resultado, lo que significa que se dirigen exclusivamente por los objetivos y resultados. Además de ser conocidos por desear feedback inmediato, también se sabe que tienen ciertos problemas aceptando las críticas, y aunque son respetuosos de la autoridad, no tienen grandes problemas en desafiarla cuando no están de acuerdo en lo que se les propone. Se sienten cómodos con el cambio, más abiertos y tolerantes, confiados y optimistas sobre su futuro, además de ser eficientes en trabajar interconectados para lograr sus objetivos, aun con gente más allá de sus fronteras nacionales. Su habilidad para maximizar su efectividad en el trabajo ha hecho que muchos los tachen de "perezosos", aunque esto puede estar más relacionado con el modelo mental de trabajo que ellos mismos tienen. En general, desean lograr un buen balance vida/trabajo, y vivir el momento, por lo que les interesa un ambiente de trabajo flexible, opuesto al horario de trabajo tradicional. Es de resaltar su alta confianza en sí mismos, devenida en narcisismo, y el hecho de que muchos se encuentran motivados en adquirir habilidades para mantenerse en buenas posiciones en el mercado laboral, por lo que están constantemente preocupados, aún luego de graduados, de sumar nuevas destrezas. Son pensadores críticos, que llegan con innovadoras y creativas ideas para ayudar. Para ellos no sólo un salario competitivo es importante, sino también un buen ambiente de trabajo. No desean estar en la misma posición por un largo tiempo, les gusta el cambio y el desarrollo, preferiblemente rápido. Son agresivos y ambiciosos en su carrera, y encuentran más importantes los desafíos para su desarrollo que un empleo de por vida, por lo que una organización será eficaz en retenerlos si les puede ofrecer múltiples oportunidades de crecimiento, aun dentro de sí misma. En pocas palabras, buscan constantemente desarrollar sus talentos y consideran cada trabajo como una oportunidad a ser añadida a sus valores. 


\section{Metodología de la investigación}

El diseño metodológico propuesto para la investigación recupera la modalidad de la triangulación metodológica, con la pretensión de alcanzar mayor certidumbre en las inferencias inductivas realizadas. En este sentido, se combinaron técnicas de recolección del enfoque cuantitativo y del diseño cualitativo. Para el primer enfoque, se diseñó y administró una encuesta auto-administrada con preguntas de opción múltiple y escalas actitudinales. El instrumento contó con 26 preguntas tendientes a confirmar, por un lado, las características psicosociales de la generación Z; y, por otro lado, a reconocer sus miradas y concepciones acerca del empleo. Se elaboró una muestra no probabilística por decisión razonada, por cuotas, de un tamaño de 400 casos, que considere al universo bajo estudio. Por cuestiones de conveniencia para el acceso a los datos, se privilegió la realización de las encuestas en el ámbito de la Universidad Argentina de la Empresa (Buenos Aires, Argentina), a aquellos estudiantes que cumplieran con el criterio de edad reconocido por la literatura (es decir, nacidos a partir de 1995). No se consideró relevante la orientación por especialidad, de modo tal que la muestra contempla diversos perfiles profesionales. La recolección de los datos fue realizada en el período de julio-agosto de 2016 . Según los usos y costumbres de la metodología cuantitativa, la encuesta fue administrada en formato papel, y luego los datos fueron codificados, tabulados y analizados a partir del sistema estadístico SPSS.

De acuerdo con la estrategia multi-método, se condujeron también entrevistas en profundidad a miembros de la generación Z (con el mismo criterio de selección que la muestra no probabilística para datos cuantitativos), con el propósito de ahondar y profundizar en las significaciones de ciertas actitudes y representaciones sobre el mundo del trabajo. Para ello, se condujeron entrevistas semi-estructuradas que recuperaron las principales dimensiones de análisis reconocidas en el problema de investigación. En total, se realizaron 12 entrevistas cualitativas. El criterio del tamaño de esta muestra intencional por decisión razonada fue la recursividad discursiva y la saturación teórica (Glaser y Strauss, 1967). La técnica de análisis de datos cualitativos fue el análisis de contenido.

\section{Resultados y principales aspectos de discusión}

El análisis de los datos cuantitativos permite concluir que la mayor parte de los encuestados privilegia el trabajo por cuenta propia. El 74\% de la muestra prefiere trabajar en un proyecto propio. En este sentido, esta actitud hacia el trabajo se condice con la característica psico-social asociada al emprendorismo propio de esta generación histórica. Este dato polemiza con lo sostenido por lorgulescu (2016), quien refiere en su investigación sobre la generación Z rumana que sólo un 11,5\% de los encuestados prefiere el trabajo independiente. En su trabajo también sostiene que el $44,9 \%$ de los encuestados preferiría trabajar en una compañía importante de tipo internacional. Estas diferencias empíricas entre el estudio conducido a nivel local y el de otros especialistas puede ser analizado a la luz de los específicos contextos socio-históricos. Es probable que el emprendorismo a nivel local sea mayormente valorado dado el contexto. En el caso argentino, se debe a varios factores, entre los cuales es posible advertir que ciertos 
desarrollos "personales" en ciertos sectores de diversas industrias han dado como resultado un enorme éxito económico a aquellos que los han llevado adelante. Rojasa y Siga (2009) explican que los emprendimientos personales se presentan como una opción más atractiva en contextos de crisis económica, dado que suponen menores exigencias legales para su inicio. Asimismo, es posible advertir una valoración positiva de la "iniciativa personal" en medios de comunicación y, fundamentalmente, en la publicidad (Rúa, 2014).

El trabajo es reconocido como una actividad importante para los encuestados en virtud de las posibilidades de beneficios económicos que el mismo habilita. El 51\% considera que el trabajo es relevante porque les permite ganar dinero, al mismo tiempo que un $34 \%$ considera que les da independencia. En todos los casos, se advierte un sesgo individualista en este tipo de orientaciones. Tal característica es reconocida como distintiva de los miembros de la Generación Z. Ese componente individualista también puede ser entendido a partir del contexto socio-histórico específico: la globalización entendida en su condición multidimensional convoca a la conformación de contextos de amplios y acelerados cambios sociales, cuyos impactos en la conformación de las subjetividades de los actores pertenecientes a la generación Z provocan características psico-sociales asociadas al individualismo y la autosatisfacción (Maioli et al, 2014).

Por otro lado, al momento de ponderar los elementos que les permitirían elegir un trabajo, un $34 \%$ considera que el criterio fundamental para establecer tal decisión es el beneficio económico. Es decir, se valora al trabajo por las posibilidades de obtener un buen salario. Al mismo tiempo, un $20 \%$ de la población encuestada sostiene que elegiría un trabajo que se encuentre vinculado con su formación académica, lo cual establece también una interesante relación entre empleo-educación. Un 15\% valoraría las condiciones de trabajo, especialmente aquellas vinculadas con el clima organizacional y las relaciones entre compañeros y jefes.

Con relación a las condiciones específicas de trabajo, un $43 \%$ de los encuestados prefiere integrarse a equipos de trabajo organizados en estructuras más horizontales. Al mismo tiempo, el 36\% de la población encuestada sostiene que preferiría desarrollar sus actividades bajo la modalidad "home office" (es decir, de manera individual). En gran medida, estas predilecciones no sólo se alinean con lo relevado por otros especialistas (Fister Gale, 2015; lorgulescu, 2016), sino que al mismo tiempo confirman ciertas características psico-sociales de los miembros de la nueva generación, especialmente su mirada individualista. Los datos recabados resultan insuficientes para refutar algunos estudios previos (Adecco, 2015) que insisten en sostener que los representantes de la generación Z privilegian el trabajo individual por encima del grupal. Si bien es cierto que los Gen Z rechazan las estructuras burocráticas tradicionales, no resulta posible afirmar de manera absoluta que rechacen los espacios de socialización que las organizaciones promueven. En tal caso, resulta cierto que, a partir de su particular apropiación de las herramientas tecnológicas, los Gen Z aceptan que las modalidades de interacción que se implican en un proceso de trabajo pueden ser conducidas de manera "mediada", es decir, sin situación de co-presencia. 
Al momento de establecer cuáles serían los factores a tener en consideración al momento de seleccionar un empleo en relación de dependencia, los miembros de la generación Z optaron por la estabilidad (56\%), la posibilidad de desarrollo profesional (42\%) y la aplicación de saberes aprendidos durante su formación (33\%). Sorprendentemente, sólo un $9 \%$ señaló que un criterio de selección al momento de elegir una compañía donde trabajar sería el paquete de beneficios que tal empresa pudiera ofrecer. En general, estas respuestas encuentran concordancia con otros estudios previos realizados (Wood, 2013; Tulgan, 2013; lorgulescu, 2016). Tal vez la única diferencia con tales estudios sea el criterio del nivel salarial. En la muestra seleccionada para este estudio, el salario como elemento de decisión, si bien resulta importante (un $34 \%$ de los encuestados lo señalaron como el criterio principal), no es el más significativo. El contexto socio-económico argentino puede ser aquí la variable que opere como explicativa: la constante sucesión de crisis económicas, y su consecuente afectación sobre las condiciones de la masa laboral como principal variable de ajuste, puede ser la condición que afecta ciertas representaciones sociales sobre el trabajo que aún tienen fuerte impacto en las subjetividades de los miembros de la Gen Z.

Al igual que el estudio conducido por lorgulescu (2016), las respuestas obtenidas por la muestra encuestada para este estudio revelan que los miembros de la Generación Z prestan poca importancia a los "beneficios tradicionales" que las compañías ofrecen a sus empleados, tales como medicina pre-paga, vacaciones pagas, e incluso condiciones de comodidad en el puesto de trabajo. De los miembros de la Gen Z encuestados, tan sólo un 5\% resaltó como significativo este paquete de beneficios.

Con relación a las habilidades que los miembros de la Gen Z deben poner en práctica en sus trabajos, la gran mayoría de los encuestados afirma que son de tipo “informáticas". El $86 \%$ de los encuestados que trabaja o ha trabajado afirma que la computadora es la herramienta primordial para la realización de sus tareas. Sin embargo, al momento de indagar sobre las habilidades informáticas que tienen los miembros de la Gen Z, un $77 \%$ afirma que no domina plenamente el uso de los principales programas informáticos que son de uso habitual en los trabajos (tales como el procesador de textos, planillas de cálculo o bases de datos). Por el contrario, una importante mayoría (92\%) señala tener un conocimiento avanzado en el uso de redes sociales (tales como Facebook, Twitter o Pinterest). En conclusión, la apropiación de la tecnología de los "nativos digitales" se encuentra más asociada al uso de redes sociales que a programas informáticos que luego pueden ser aplicados en sus puestos de trabajo. En línea con el estudio de lorgulescu (2016), los encuestados para este estudio resaltaron el hecho de contar con "habilidades soft" para el desempeño de sus tareas (tales como proactividad, responsabilidad y productividad).

\section{Conclusiones}

Los miembros de la Generación Z cuentan con características psico-sociales específicas que los diferencian de las generaciones históricas previas. Asimismo, se encuentran próximos a su incorporación al mercado de trabajo, lo cual exige, en gran medida, una reflexión en torno a sus peculiaridades y al modo en que las estructuras 
organizacionales se encuentran en condiciones de recibirlos. Fister Gale (2015) expresa que las organizaciones deben conocer en profundidad las expectativas de los Gen Z para llevar adelante exitosos procesos de reclutamiento de estos nuevos talentos, al tiempo que adecuar sus ofertas de beneficios para poder retenerlos. Aún al día de hoy perduran ciertas representaciones sociales en relación con lo que esperan las nuevas generaciones sobre sus posiciones laborales en las organizaciones que no siempre encuentran un correlato con la realidad.

Esta investigación empírica pretende echar luz sobre algunas de las características psicosociales más significativas de la Gen Z. Al mismo tiempo, pretende reconocer que muchas de tales características inciden directamente en el modo en el cual estas generaciones se incorporarán plenamente en el mercado de trabajo. En virtud de ello, el estudio confirma en gran medida algunos hallazgos previos (Iorgulescu, 2016) con relación a los patrones de desarrollo esperados por los miembros de la Gen Z. Estos jóvenes esperan del trabajo al menos tres cuestiones fundamentales: seguridad económica (por medio de buenos salarios), acompañamiento en los procesos de incorporación a las estructuras organizacionales (por medio de procesos de inducción bajo la modalidad de mentores) y flexibilidad horaria (por medio de modalidades de trabajo orientadas a objetivos).

Estas cuestiones, claramente, obligan a las estructuras organizacionales a llevar adelante procesos de reorganización de sus modalidades tradicionales de disposición del trabajo. La estructuración de equipos de trabajo con formas de autoridad más bien horizontales es uno de los principales desafíos para las organizaciones. Al mismo tiempo, las empresas no deben dar por supuesto que la condición de nativos digitales de la Gen Z presupone un amplio dominio de los recursos informáticos tradicionales (como ser procesador de textos, sistemas de presentaciones, etc.). La apropiación de la tecnología de los miembros de la Generación Z parece ser algo más superficial de lo que uno podría presuponer. Por ello, resulta valioso que las organizaciones dispongan de sólidos programas de capacitación y entrenamiento en el uso de los sistemas informáticos necesarios para el desempeño de las tareas.

Asimismo, en línea con lo afirmado por Fister Gale (2015), las organizaciones deben disponer de procesos de selección orientados a reconocer los saberes previos de los miembros de la Generación Z (especialmente aquellos obtenidos por medio de la educación formal universitaria), dado que estos jóvenes esperan que los empleos puedan ser el espacio en donde puedan aplicar sus conocimientos. Un factor de frustración (asociado a la alta rotación de los jóvenes) se advierte cuando no hay coincidencia entre lo que la organización propone y lo que los jóvenes esperan. El estudio conducido revela que los jóvenes de la Gen Z esperan poner en práctica sus saberes adquiridos, y que tal aplicación implica un "plan de carrera". Los jóvenes de la Gen Z no buscan necesariamente reconocimiento por medio de designaciones formales de cargos (Iorgulescu, 2016), sino más bien la realización de actividades dentro de la organización que se vinculen fuertemente con sus saberes aprendidos. 
Un elemento que ha generado amplia discusión entre los especialistas (Adecco, 2015; Half, 2015) refiere a los paquetes de beneficios que deben ser ofrecidos por parte de las organizaciones para convocar a los miembros de las nuevas generaciones, especialmente a los Z. Durante bastante tiempo se consideró que mejores condiciones del sitio de trabajo era una demanda asociada con los jóvenes, especialmente con relación a actividades lúdicas o de esparcimiento. El estudio revela que el salario aún es un criterio fundamental por parte de los representantes de esta generación; al mismo tiempo, la flexibilidad horaria sería el segundo factor más importante al momento de aceptar (y permanecer) en una organización. Por último, cuestiones asociadas al clima organizacional también se encontrarían dentro de los factores que los miembros de la Generación Z evaluarían al momento de elegir un empleo.

El estudio realizado cuenta con limitaciones metodológicas (fundadas principalmente en la realización de una muestra intencional no probabilística) que limita sus hallazgos a la muestra seleccionada. No obstante, sus resultados encuentran confirmación en estudios previos. Asimismo, plantea la necesidad de establecer líneas de investigación futura que puedan servir de un fundamento más sólido para elaborar conclusiones asociadas al problema de investigación. En virtud de ello, se sugiere que otros investigadores propongan la realización de muestras probabilísticas de mayor envergadura que pudieran confirmar las intuiciones presentes en este trabajo. De igual modo, una línea de investigación fructífera sugerida en este trabajo se asocia a la necesidad de conocer con mayor profundidad las expectativas de las estructuras organizacionales y los modos de adaptación que necesariamente deben asumir a los efectos de poder incorporar y retener a los jóvenes talentos de la Gen Z.

\section{Referencias Bibliográficas}

ADDOR, M. L. (2011). Generation Z: What is the Future of Stakeholder Engagement? Institute for EMERGING ISSUES NC State University, pp. 1- 7. Documento electrónico en línea, disponible en https://iei.ncsu.edu/wpcontent/uploads/2013/01/GenZStakeholders2.pdf. Fecha de acceso: 23-09-2016.

ADECCO (2015). Generation Z vs. Millennials. Documento electrónico en línea: disponible en http://pages.adeccousa.com/rs/107-IXF 39/images/generation-z-vs-millennials.pdf, fecha de acceso: 15-09-2016.

BENNETT, J., PITT, M., PRICE, S. (2012). Understanding the impact of generational issues in the workplace. Facilities, Vol. 30 (Iss. 7/8), pp. 278-288.

CHERRINGTON, Ruth (1997) Generational Issues in China: A Case Study of the 1980s Generation of Young Intellectuals, en The British Journal of Sociology, Vol. 48, No. 2 (Jun., 1997), pp. 302-320.

FISTER Gale, S. (2015) Forget Millenials: Are you ready for Generation Z? Chief Learning Officer. Documento electrónico en línea, disponible en www.CLOmedia.com, fecha de acceso: 23-08-2016. 
HALF, R. (2015). Get ready for generation Z. Documento electrónico en línea, disponible en: https://www.roberthalf.com/sites/default/files/Media_Root/images/rhpdfs/rh_0715_wp_genz_nam_eng_sec.pf, Fecha de acceso: 22-09-2016.

IORGULESCU, M. C. (2016) Generation Z and its perception of work. Cross-Cultural Management Journal. Volume XVIII, Issue 1 (9).

MAIOLI, E. et al (2014) Generación Z, Educación y Empleo. Una revisión de elementos conceptuales para el estudio de los impactos generacionales en el ámbito institucional de la educación y el empleo en Argentina (2013). Ponencia presentada en XX Jornadas de Investigación y IX Encuentro de Investigadores en Psicología del MERCOSUR, Buenos Aires, 2014.

PILCHER, J. (1994). Mannheim's sociology of generations: an undervalued legacy. BJS, Vol. 45, No. 3, (September 1994), pp. 481-495.

ROJASA, G. M. y SIGA, L. (2009). On the nature of micro-entrepeneurship: evidence from Argentina. Applied Economics, 41 (21), 2667-2680.

RÚA, M. (2014) Yo, entrepreneur. Documento electrónico en línea, disponible en:

http://www.lanacion.com.ar/1671916-yo-entrepreneur.Fecha de acceso: 11-09-2016.

SMOLA, Karen Wey and SUTTON, Charlotte D. (2002) Generational Differences: Revisiting Generational Work Values for the New Millennium en Journal of Organizational Behavior, Vol. 23, No. 4, Special Issue: Brave New Workplace: Organizational Behavior in the Electronic Age (Jun., 2002), pp. 363-382.

TULGAN, B. (2013). Meet Generation Z: The second generation within the giant "Millennial" cohort. RainmakerThinking. Documento electrónico en línea, disponible en:

http://rainmakerthinking.com/assets/uploads/2013/10/Gen-Z-hitepaper.pdf. Fecha de acceso: 03-08-2016.

WOOD, S. (2013). Generation Z as Consumers: Trends and Innovation. Institute for EMERGING ISSUES: NC State University, pp., 1-3. Documento electrónico en línea, disponible en:

https://iei.ncsu.edu/wpcontent/uploads/2013/01/GenZConsumers.pdf, Fecha de acceso: 03-08-2016. 